\title{
Frequency Analysis of Transient Light Transport with Applications in Bare Sensor Imaging
}

\author{
Di Wu ${ }^{1,2,5 \star}$, Gordon Wetzstein ${ }^{1}$, Christopher Barsi ${ }^{1}$, Thomas Willwacher ${ }^{3}$, \\ Matthew O'Toole ${ }^{4}$, Nikhil Naik ${ }^{1}$, Qionghai Dai ${ }^{2}$, \\ Kyros Kutulakos ${ }^{4}$, and Ramesh Raskar ${ }^{1}$ \\ ${ }^{1}$ MIT Media Lab, ${ }^{2}$ Department of Automation, Tsinghua University, \\ ${ }^{3}$ Department of Mathematics, Harvard University, ${ }^{4}$ Department of Computer Science, \\ University of Toronto, ${ }^{5}$ Graduate School at Shenzhen, Tsinghua University
}

\begin{abstract}
Light transport has been analyzed extensively, in both the primal domain and the frequency domain; the latter provides intuition of effects introduced by free space propagation and by optical elements, and allows for optimal designs of computational cameras for tailored, efficient information capture. Here, we relax the common assumption that the speed of light is infinite and analyze free space propagation in the frequency domain considering spatial, temporal, and angular light variation. Using this analysis, we derive analytic expressions for crossdimensional information transfer and show how this can be exploited for designing a new, time-resolved bare sensor imaging system.
\end{abstract}

Key words: Light transport, Fourier analysis, Time of flight, Lensless imaging

\section{Introduction}

Some of the fastest electronic recording devices include the Edgerton stroboscope [1], which can record events at one million frames per second. Today, ultrafast sensors (e.g. [2]) can produce visual information at effectively one trillion frames per second, roughly one million times faster than Edgerton's electronic strobes. This technology allows for the capture of light as it propagates through space and facilitates a wide variety of applications in computer vision. Indeed, such high time resolution makes available to computer vision applications a new degree of freedom, namely, time-of-flight of light propagation.

Starting from first principles, we analyze light propagation in free space in the primal and the frequency domains, considering space, angle, and time for a finite speed of light. We discuss cross-dimensional information transfer and derive upper bounds for ultra-fast streak sensors, which record only subsets of the high-dimensional space. Based on this analysis, we propose a new imaging technique that does not require any optical elements besides a time-resolved sensor and a pulsed light source. Summarized in Fig. 1, our contributions are as follows:

\footnotetext{
^ This work was completed while Di Wu was a visiting student at the MIT Media Lab.
} 


\begin{tabular}{|r|c|c|c|}
\hline & space-angle $x-\nu$ & space-time $x-t$ & space-angle-time $x-\nu-t$ \\
\hline $\begin{array}{r}\text { forward propagation } \\
\text { (primal domain) }\end{array}$ & shear in $x$ & $\begin{array}{c}\text { convolution with } \\
\text { depth-dependent hyperbola }\end{array}$ & $\begin{array}{c}\text { shear in } x \\
\text { hyperbolic curvature in } t\end{array}$ \\
\hline $\begin{array}{r}\text { forward propagation } \\
\text { (frequency domain) }\end{array}$ & shear in $f_{\nu}$ & Fourier transform of hyperbola & convolution in $f_{\nu}$ \\
\hline $\begin{array}{r}\text { inverse propagation } \\
\text { (primal domain) }\end{array}$ & shear back in $x$ & $\begin{array}{c}\text { single depth: deconvolution } \\
\text { multiple depths: tomography }\end{array}$ & tomographic reconstruction \\
\hline $\begin{array}{r}\text { inverse propagation } \\
\text { (frequency domain) }\end{array}$ & shear back in $f_{\nu}$ & $\begin{array}{c}\text { single depth: division } \\
\text { multiple: filtered backprojection }\end{array}$ & filtered backprojection \\
\hline
\end{tabular}

Fig. 1. Overview of operators for forward and inverse light field transport. While conventional analysis (e.g. [3]) considering only time-independent transport (left column), we analyze time-resolved forward and inverse light transport in this paper (center and right columns) and show that the operations required to inverse-propagate a light field backwards in space, time, and angle correspond to computed tomographic reconstructions.

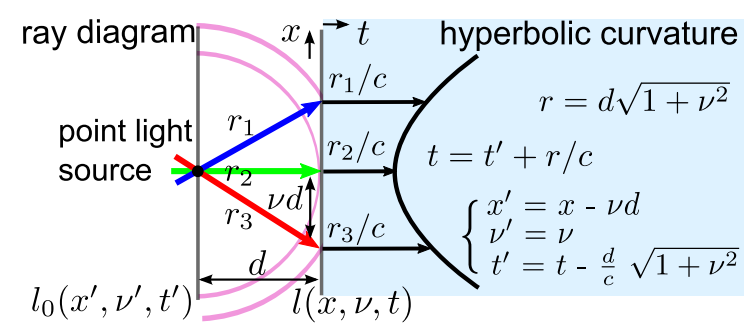

Fig. 2. Ray diagram illustrating the two-plane parameterization of light fields. Rays arrive at a plane a distance $d$ from the source at times that depend on their angle.

- We analyze free space light propagation in the frequency domain considering spatial, temporal, and angular light variation. We show that the propagation in frequency space can be modeled analytically as a combination of a shear in the light field and a convolution along the angular frequencies (Sec. 3).

- We show propagation in free space has unique information-preserving properties by transferring information among the dimensions. We derive upper bounds for how much of the information contained in one dimension is retained when capturing only the others (Sec. 4).

- Based on this analysis, we introduce a novel, bare sensor imager (Sec. 5). This approach exploits ultra-fast imaging combined with iterative reconstruction, while removing the need for optical elements, e.g. lenses or masks.

- We demonstrate, using synthetic scenes and an experimental prototype camera, the practicality of the proposed approach (Sec. 6).

\section{Related Work}

Frequency analysis of light transport [3] has been of critical importance in computer vision and graphics in evaluating the performance of imaging systems and 
identifying novel computational camera designs [4-6] or implementing faster [7] and better [8] computational processing schemes. Time-resolved forward and inverse transport has begun to be studied only recently [9].

Lensless imagers include attenuating layers, control of transmittance in space and time [10], template-based approaches using micro sensors [11], Fresnel zone plates or photon sieve for imaging using diffractive optics [12], and angle-sensitive pixels that require no off-chip optics [13]. Our approach requires only a single, high-speed sensor and does not require additional attenuating or diffractive optical elements.

Sensors with the ability to measure temporal impulse responses provide a rich source of imaging information in seismology, sonology, and optics. Reflection seismology and medical ultrasound use such measurements for performing noninvasive volumetric imaging, whereas sonar, radar, and LiDAR (light detection and ranging) technologies determine an objects range, among other properties, from the echos of sound, radio waves, and light pulses reflecting off a target object [14]. Unlike LiDAR, which measures the time of arrival of direct reflections of light, our ultra-fast sensor exploits the full time-of-flight profile, measuring photons as they arrive at the sensor over an extended period of time, for imaging both albedo and depth.

Imaging at speeds fast enough to observe radiometric changes over time has unveiled many new applications for analyzing scenes that cannot be performed with a standard camera. These ultra-fast cameras have the ability to reconstruct geometry (though not its albedo) of occluded objects "around a corner" [15], acquire in-the-wild BRDFs without using encircling equipment for scenes with known geometry [16], recover an occluded target's motion using multipath analysis [17], and decompose global light transport [18]. Our analysis of light propagation in free space produces an intuitive explanation of forward light transport within all these various works, and provides the fundamental tools for developing and understanding new ultra-fast imaging applications.

\section{$3 \quad$ Frequency Analysis of Transient Light Transport}

In this section, we analyze time-resolved light transport in free space. While an analysis of steady-state light transport is intuitive in revealing where information is localized in the frequency domain [3,5], considering the time-resolved case has, to the best knowledge of the authors, not been discussed in the computer vision literature.

Starting from first principles, space-angle light transport is commonly denoted as $[19,20]$

$$
l^{(S)}(x, \nu)=l_{0}^{(S)}(x-\nu d, \nu),
$$

where $l^{(S)}(x, \nu)$ is the light field after transport by a distance $d, x$ is the spatial variable, and $\nu$ is the relative coordinate on a plane at unit distance [20] (Fig. 2 (left)). Including the speed of light, we recognize that different ray paths arrive at different times. In order to accurately model such systems, the time-resolved 


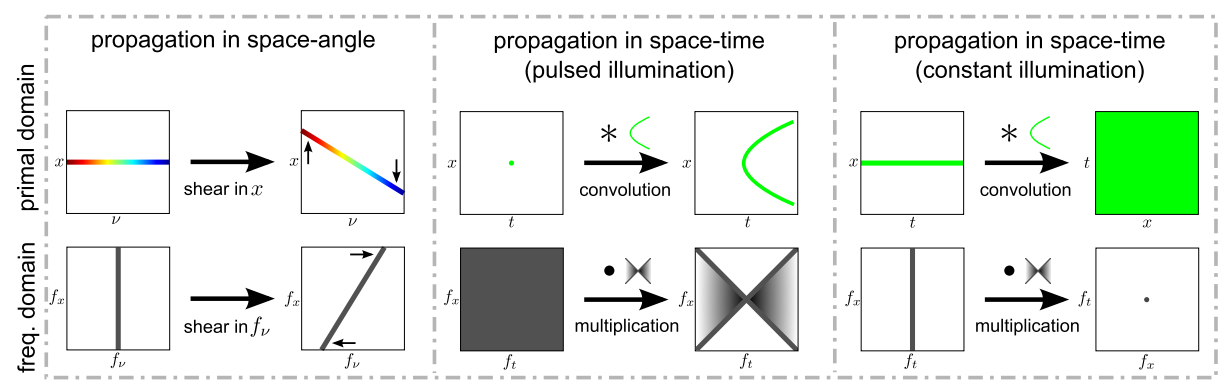

Fig. 3. Without time resolution, free space propagation shears the light field in both the primal and frequency domains (left). Due to relative time delays of different rays, a pulsed point source produces a space-time hyperbola, which acts as the convolution kernel for time-resolved light fields (center). In frequency space, this hyperbolic convolution is transformed to multiplication with its spectrum. Importantly, without a time-resolved source, only $D C$ components are propagated (right). (Note: space-angle shear is maintained, though not shown, in the time-resolved panels.)

forward light transport must consider the temporal dimension $t$, incorporating the finite speed of light $c$ as

$$
l(x, \nu, t)=l_{0}\left(x-\nu d, \nu, t-\frac{d}{c} \sqrt{1+\nu^{2}}\right)
$$

Physically, rays propating at large angles arrive later than low-angled ones. The delay can be calculated geometrically (Fig. 2). Note, e.g., for a pulsed point source, $l_{0}(x, \nu, t)=\delta(x) \delta(t)$, which, after a propagation distance $d$, becomes $l(x, \nu, t)=\delta(x-\nu d) \delta\left(t-(d / c) \sqrt{1+\nu^{2}}\right) \sim \delta(x / d-\nu) \delta\left(t-(d / c) \sqrt{1+(x / d)^{2}}\right)$. From the second $\delta$-factor, we see that time-resolved propagation produces a hyperbolic space-time curvature (Fig. 2, right). A summary of space-time propagation is shown in Fig. 3.

In the Fourier domain, propagation in free space for the steady-state case is a shear along the angular frequencies [3]:

$$
\tilde{l}^{(S)}\left(f_{x}, f_{\nu}\right)=\tilde{l}_{0}^{(S)}\left(f_{x}, f_{\nu}+f_{x} d\right)
$$

where $\sim$ denotes the Fourier transform of a quantity and $f_{x}, f_{\nu}$ are the frequency variables of space and angle, respectively. For this case, the spaceangle shear in the primal domain (Eq. 1) intuitively translates to a shear along the opposite dimension in the frequency domain. As illustrated in Fig. 4, the time-resolved light field spectrum remains sheared in the $f_{x^{-}} f_{\nu}$ dimension, but is now blurred in the $f_{t}$ dimension, mainly along two branches that form an $x$-shape. To quantify this result, we calculate the Fourier transform of the time resolved light field after propagation in free space (both Eq. 4 and Eq. 5 are 

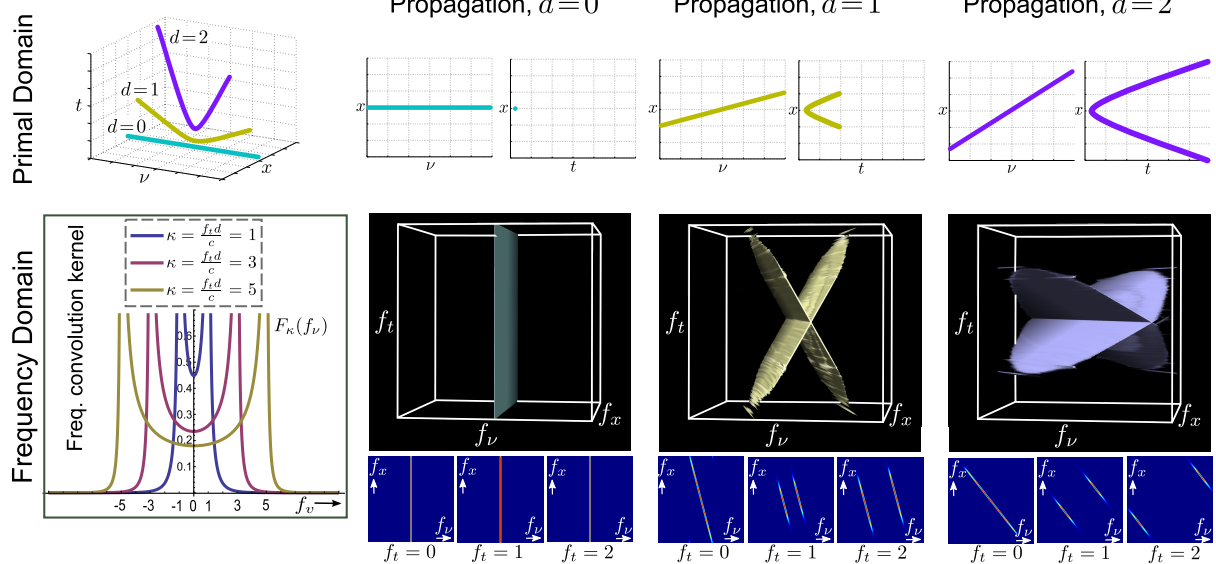

Fig. 4. Visualization of light field propagation. Top row: primal domain propagation of light pulse $\left(l_{0}(x, \nu t)=\delta(x) \delta(t)\right)$. As the propagation distance $d$ increases, the $x$ - $t$ hyperbola increases in curvature, and the $x-\nu$ shear becomes steeper. Bottom row: corresponding frequency domain representations. The steady state $\left(f_{t}=0\right)$ cross section is the standard Fourier shear, which splits into an $\times$-shape off-plane. (Left): cross section of spectrum at various off-axis positions.

derived in the supplement):

$$
\begin{aligned}
\tilde{l}\left(f_{x}, f_{\nu}, f_{t}\right) & =\iiint e^{-2 i \pi f_{x} x-2 i \pi f_{\nu} \nu-2 i \pi f_{t} t} l_{0}\left(x-\nu d, \nu, t-\frac{d}{c} \sqrt{1+\nu^{2}}\right) d x d \nu d t \\
& =\tilde{l}_{0}\left(f_{x}, f_{\nu}+f_{x} d, f_{t}\right) \underset{f_{\nu}}{*} F_{\frac{f_{t} d}{c}}\left(f_{\nu}\right),
\end{aligned}
$$

where

$F_{\kappa}\left(f_{\nu}\right):=\int d \nu e^{-2 i \pi f_{\nu}-2 i \pi \kappa \sqrt{1+\nu^{2}}}= \begin{cases}-\frac{\pi i \kappa}{\sqrt{\kappa^{2}-f_{\nu}^{2}}} H_{1}^{(2)}\left(2 \pi \sqrt{\kappa^{2}-f_{\nu}^{2}}\right) & \text { for } \kappa^{2}>f_{\nu}^{2} \\ \frac{2 i \kappa}{\sqrt{f_{\nu}^{2}-\kappa^{2}}} K_{1}\left(2 \pi \sqrt{f_{\nu}^{2}-\kappa^{2}}\right) & \text { for } \kappa^{2}<f_{\nu}^{2}\end{cases}$

Here, $H_{1}^{(2)}$ is a Hankel function of the second kind, $K_{1}$ is a modified Bessel function of second kind, and $\kappa=f_{t} d / c$. Cross sections of this function are plotted in Fig. 4. Eq. 4 mathematically expresses the effects of propagation on the light field spectrum through 1) shearing the space-angle dimension, then 2) convolving the result with $F_{\underline{f_{t} d}}\left(f_{\nu}\right)$ along the $f_{\nu}$ dimension.

Note in particular that as $\kappa \rightarrow 0$, the kernel approaches the delta function for all $f_{\nu}$, so that the final propagated light field approaches the time-independent case. This simplification occurs for either $d$ or $f_{t}$ vanishing (corresponding to no propagation or considering only the steady-state component $f_{t}=0$, respectively), or for the speed of light $c$ going to infinity (corresponding to neglect of time-resolution). With the above assumptions, the system (Eq. 4) reduces to the commonly used steady-state Fourier propagator (Eq. 3) (derived in the Supplement). 
The convolution operation generally "blurs" information in the convolved dimension. We analyze this behavior in more detail in Sec. 4 and demonstrate in Sec. 5 that this can be directly exploited to design a novel imaging system. Here, however, the kernel profile depends on not only the convolved dimension, but also the parameter $f_{t} d / c$. As shown from Eq. 5, there are two regimes to consider. Whereas the decay of $F_{\kappa}$ is exponential for $|\kappa|<\left|f_{\nu}\right|$ (so that information located there cannot be detected to first order), the falloff is much slower for $|\kappa|>\left|f_{\nu}\right|$ (where infromation can be detected). At the boundary, $\kappa=f_{\nu}$, and $F_{\kappa}$ becomes singular. We call this boundary the "light cone" ${ }^{1 "}$ and use it to distinguish between these two regimes. Light propagation of a point light source in the primal and frequency domain is illustrated in Fig. 4.

\section{Cross-dimensional Information Transfer and Space-Time-Angle Bandwidth Analysis}

The Fourier analysis in the previous section shows that there is a transfer of information between the different dimensions; space, time, and angle are all coupled. We exploit this information transfer by proposing an ultra-fast lensless sensor that captures only space and time; the recorded data, called a streak image $s(x, t)$, contains angular information that can be used to infer image and depth of the scene. To analyze the bandwidth and reconstruction limits, we write $s(x, t)$ (Fig. 2 (right)) as:

$$
s(x, t)=\int l(x, \nu, t) d \nu=\int l_{0}\left(x-\nu d, \nu, t-\frac{d}{c} \sqrt{1+\nu^{2}}\right) d \nu
$$

Equivalently, the streak spectrum is found by setting the $D C$ component $\left(f_{\nu}\right)$ to zero.

To simplify the analysis, we consider a Lambertian scene, so that the light field becomes $l_{0}(x, \nu, t)=l_{0}^{L}(x, t)$, i.e., it is angle-independent. Using (Eq. 4), we can write the measured streak image as (derived in the Supplement):

$$
\tilde{s}\left(f_{x}, f_{t}\right)=\tilde{l}_{0}^{L}\left(f_{x}, f_{t}\right) F_{\frac{f_{t} d}{c}}\left(f_{x} d\right)
$$

Physically, the final spectrum is the original light field spectrum multiplied by a space-time-dependent weighting factor (or MTF), whose characteristic width is determined by the propagation distance $d$. Thus, the kernel $F_{\kappa}$ acts as a modulation transfer function. This equation allows us to derive an upper bound on the spatial frequencies $f_{x}$ of a Lambertian plane at distance $d$ that can be recovered from a streak image $s(x, t)$.

To gain some intuition, consider the simplest case of a cosine albedo (of spatial frequency $f_{0}$ ) that is illuminated by a planar impulse: $l_{0}^{L}(x, t)=$ $\cos \left(2 \pi f_{0} x\right) \delta(t)$, as shown in Fig. 5. (Experimentally, this setup could be effected by illuminating the object with a pulsed point source whose origin is far from the

\footnotetext{
1 The concept of light cones is commonly used in space-time physics, see e.g. www . phy.syr.edu/courses/modules/LIGHTCONE/minkowski.html
} 
object so that the object sees an approximately planer wavefront.) Immediately after the mask, the Fourier transform is a pair of vertical delta functions, centered at $\pm f_{0}$. After propagation, the spectrum is multiplied by the MTF above. For $c\left|f_{x}\right|>\left|f_{t}\right|, F_{\frac{f_{t} d}{c}}\left(f_{x} d\right)$ decays exponentially, so that, to first order, information in these regions is lost. For $c\left|f_{x}\right|<\left|f_{t}\right|$, the information is preserved. This behavior is shown in Fig. 5 (top right).

In order to detect the signal, its space-time bandwidth must lie within the camera's. Because there is no cross-dimensional information transfer, i.e., spatial frequencies are not sheared into time, the resolution criteria is the same as for conventional imaging: the detector's spatial bandwidth $f_{x}^{\max }$ must exceed the signal frequency: $f_{0}<f_{x}^{\max }$, regardless of the time bandwidth.

In contrast, consider the same scene illuminated by a spherical wavefront pulse. For simplicity, we assume the point source is located on the $z$-axis a distance $d_{s}$ from the scene (Fig. 5). The initial light field becomes $l_{0}^{L}(x, t)=$ $\cos \left(2 \pi f_{0} x\right) \delta\left(t-\left(d_{s} / c\right) \sqrt{1+\left(x / d_{s}\right)^{2}}\right)$. Now, the light field's Fourier transform is the convolution of the transforms of the factors. After propagating a distance $d_{r}$, the resulting streak spectrum (derived in the supplement) is

$$
\tilde{s}\left(f_{x}, f_{t}\right)=F_{\frac{f_{t} Z}{c}}\left(Z\left(f_{x}+d_{s} f_{0} / Z\right)\right)+F_{\frac{f_{t} Z}{c}}\left(Z\left(f_{x}-d_{s} f_{0} / Z\right)\right) .
$$

where $Z \equiv d_{s}+d_{r}$. When plotted in the $f_{x^{-}} f_{t}$ plane (see Fig. 5 , bottom), we see a strong signal in the time domain (i.e., along the vertical axis), as we would expect from a spherical pulse, which illumantes different scene points at different times. In this case, the scene frequency is recovered if $f_{t}^{\max }$ is large enough (roughly $f_{t}^{\max }>\frac{d_{s} c}{Z} f_{0}$ ), regardless of the spatial bandwidth. Thus, a sufficiently fast camera can super-resolve object features.

Note that in the limiting case of $f_{t}^{\max } \rightarrow 0$, the detector no longer detects time-resolution. In this case, one will measure a signal only if its space bandwidth is greater than the signal's bandwidth and if the propagation distance is sufficiently small so as to have the signal fall within $\sim Z^{-1}$ of the propagator singularity. These are the same criteria for convention lensless imaging setups. Using these considerations, we expect that time-resolved measurements can be used to extract scene information otherwise unavailable.

\section{Bare Sensor Imaging using Inverse Light Transport}

While the previous sections analyzed time-resolved forward light propagation and the cross-dimensional correlations, including upper bounds on preserved information, we now proceed with the inverse problem. Given a streak image, that is space-time scene information, captured with a bare sensor with no additional optical elements, such as lenses or attenuators, we aim at reconstructing image and depth of the scene. This is an ambitious goal; conventional cameras can only resolve a two-dimensional image without any depth information. A light field camera, recording space and angle $x-\nu$, provides sufficient information to refocus the photograph in post-processing [7]; unfortunately, this usually comes 


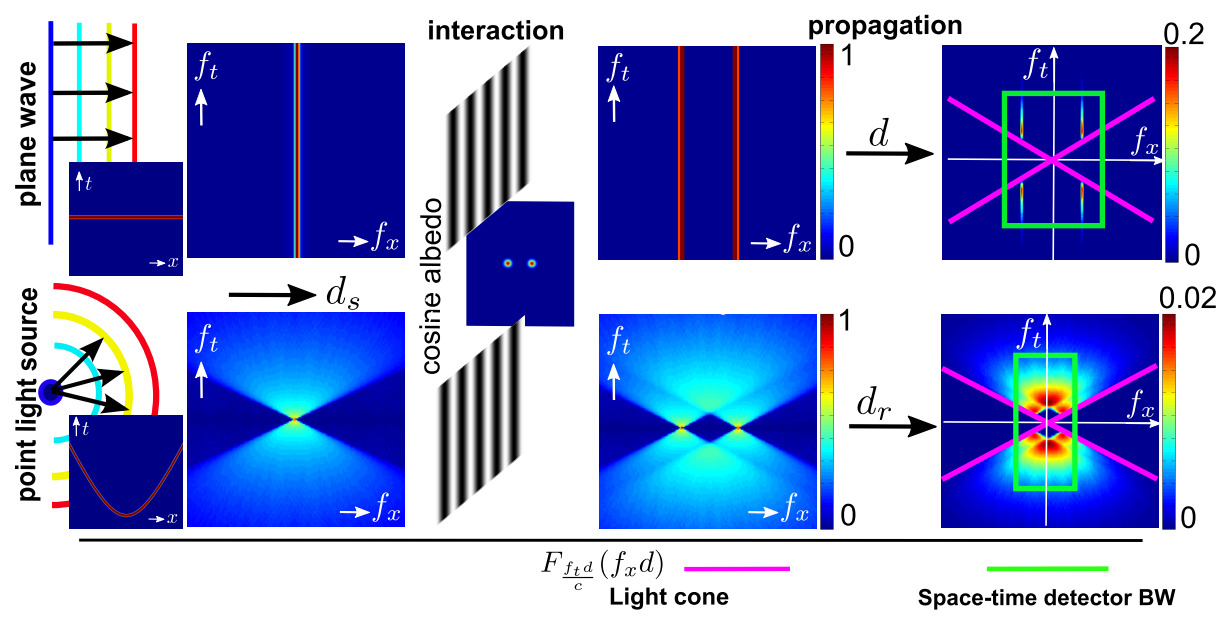

Fig. 5. Time-resolved bandwidth analysis of a Lambertian scene of single spatial frequency $f_{0}$. Top: planar impulse illumination. Immediately after interaction, the spectrum copies the incident spectrum at $\pm f_{0}$. After propagation, the signal is attenuated along the $f_{x}$ direction, but is preserved in time. The green line indicates the space-time bandwidth of the sensor, and the pink line represents the propagation MTF light cone (slopes are $\pm c$ ). Here, spatial resolution must be greater than $f_{0}$ for detection. Bottom row: point pulse illumination. After propagation, significant information is located along the $f_{t}$ direction, so that spatial bandwidth can be relaxed, e.g., information can be detected even if $f_{\max }=0$.

at the cost of a significant drop in spatial image resolution due to the employed lenslet arrays. As derived in the previous section, even a low-resolution bare sensor is theoretically capable of resolving image information with a higher spatial resolution, i.e., with super-resolution. For this application, we make the following assumptions for the remainder of this section: a Lambertian scene, exhibiting no significant global illumination effects or occlusions, is illuminated with a pulsed point light source and recorded with a bare sensor that provides a temporal resolving power in the lower picosecond range. Under these conditions, the timeresolved rendering equation $[21,22]$ can be written as

$$
s(\mathbf{x}, t)=I_{0} \int \frac{1}{\left(r_{s} r_{l}\right)^{2}} \rho\left(\mathbf{x}^{\prime}\right) \cos \theta_{s} \cos \theta_{l} \delta\left(t-\frac{r_{s}+r_{l}}{c}\right) d \mathbf{x}^{\prime},
$$

where the recorded streak image $s$ is restricted in space $\mathbf{x}=\left(x_{x}, x_{y}, x_{z}\right)^{T}$ to the plane $\left(x_{x}, x_{y}, 0\right)^{T}$ and $\rho(\mathbf{x})$ describes the diffuse albedo of a point. As illustrated in Fig. 6, the distances between scene point and pixel as well as light source are $r_{s}$ and $r_{l}$, respectively. The cosine terms model the angle between surface point normal and incoming or outgoing light direction, whereas the $\delta$ function describes the time-dependency of the system introduced by the pulsed point light source. Eq. 9 is a tomographic reconstruction problem [23], as each pixel in the streak image measures a weighted integral over the unknown surface albedos in 

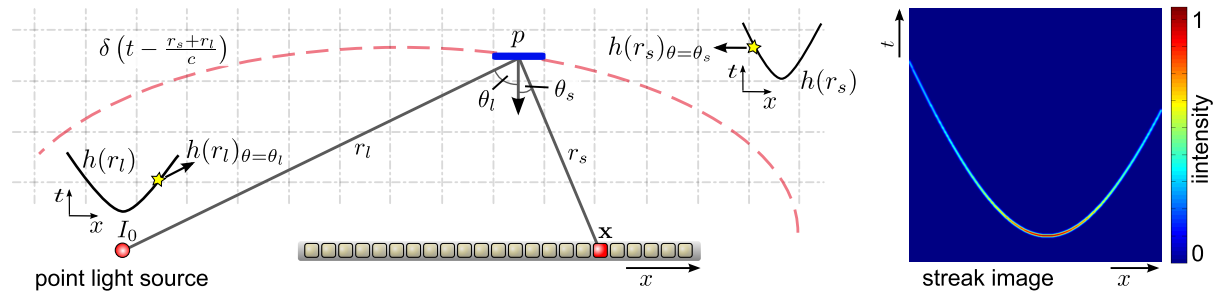

Fig. 6. One-dimensional illustration of tomographic scene reconstruction. A lensless streak sensor (center) records the time of arrival of photons emitted by a pulsed point light source. The captured streak image for a single white patch in the scene is illustrated on the right. For an unknown scene, however, the contribution at each sensor pixel $(x, t)$ is ambiguous as it may contain contributions from any point in the scene that is located along an ellipse, whose locus of points have idential path lengths from source to detector, as illustrated by the dashed red line (left).

the scene. As opposed to conventional line tomography, the integration surfaces in this application are elliptical surfaces as illustrated by the dashed red line in Fig. 6. This is similar to range tomography in seismic imaging [14] with the significant difference that no phase information is captured in our application, making the inversion problem more difficult. While filtered backprojection [23] is the most straightforward method to inverting tomographic problems, the finite sensor size in the proposed setup actually turns this to a limited-baseline tomography problem, for which backprojection is not well-defined. Instead, we employ iterative reconstruction methods [24] (see Sec. 6.1).

For this purpose, we model the scene containing an unknown geometry and surface albedos as a basis expansion:

$$
\rho(\mathbf{x})=\sum_{k=1}^{N} \rho_{k} \phi_{k}(\mathbf{x})
$$

where $\rho_{k}$ are the coefficients, and the basis functions $\phi_{k}(\mathbf{x}), k \in 1 \ldots N$. This notation allows us to write Eq. 9 as

$$
s(\mathbf{x}, t)=I_{0} \sum_{k=1}^{N} \rho_{k} \int \frac{1}{\left(r_{s} r_{l}\right)^{2}} \phi_{k}\left(\mathbf{x}^{\prime}\right) \cos \theta_{s} \cos \theta_{l} \delta\left(t-\frac{r_{s}+r_{l}}{c}\right) d \mathbf{x}^{\prime} .
$$

In practice, this formulation is discretized into a linear system $\mathbf{s}=\mathbf{P} \rho$, where each element of $\mathbf{s}$ is,

$$
s_{x t}=\sum_{k=1}^{N} \rho_{k} P_{x t}^{(k)},
$$

and we approximate the elements of matrix $\mathbf{P}$ as

$$
P_{x t}^{(k)}=\frac{I_{0}}{\left(r_{x k} r_{l k}\right)^{2}} d A_{k} \cos \theta_{x k} \cos \theta_{l k} \zeta_{t}\left(\frac{r_{x k}+r_{l k}}{c}\right) .
$$



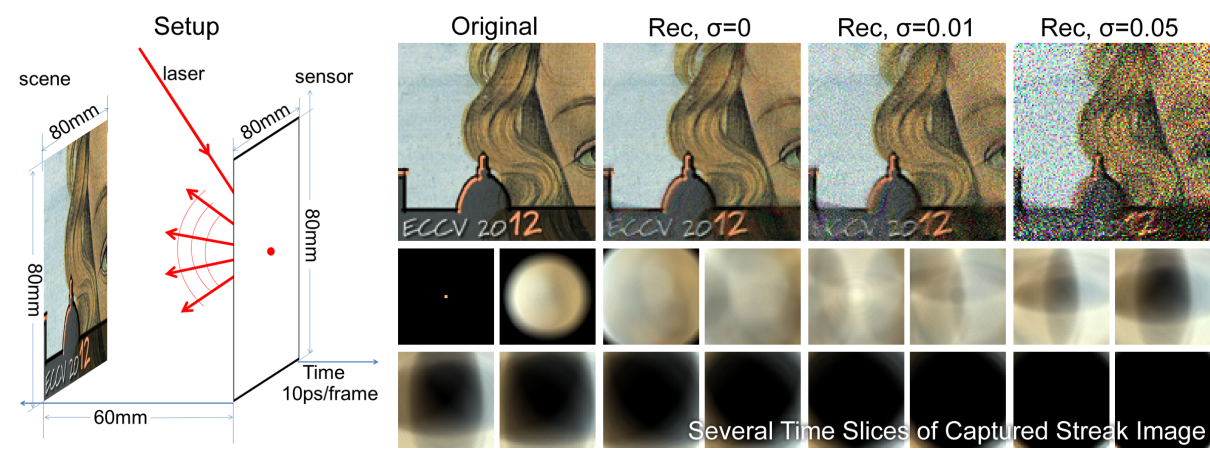

Fig. 7. Simulated reconstructions of a textured plane at a distance to the sensor. A bare sensor placed at a distance to the scene (left) captures a streak image, which is a series of $2 \mathrm{D}$ images (bottom row). The scene is reconstructed using computed tomography for varying levels of additive sensor noise (top row).

Inspired by surflets [25], we model the unknown scene geometry as a collection of planar patches of size $d A_{k}$, facing the bare sensor. The distance from patch $k$ to sensor pixel $x$ is $r_{x k}, r_{l k}$ is the distance from patch $k$ to the light source, and $\zeta_{t}(\cdot)$ maps the travel time of a photon to the nearest time slot in the streak image. Using this formulation, the final optimization problem is formulated as

$$
\underset{\rho}{\arg \min }\|\mathbf{s}-\mathbf{P} \rho\|_{2}^{2}, \text { for } 0 \leq \rho \leq 1,
$$

which can be solved with standard approaches of constrained linear optimization as discussed in Sec. 6.1.

\section{Experimental Results}

In this section, we show three different experiments of the proposed bare sensor imaging system. The results in Fig. 7 and 8 simulate a streak sensor that captures a three-dimensional volume containing two spatial dimensions as well as temporal variations. The experiments show reconstructions of only the diffuse albedos for a single plane at a distance to the sensor in Fig. 7 and for two planes at different distances in Fig. 8. In the latter case, the depth is estimated along with the diffuse albedos. Fig. 9 shows reconstructions of a simple two-dimensional scene patch from a streak sensor only measuring one spatial dimension along with temporal variation.

The results illustrated in Fig. 7 are captured with a simulated streak sensor that has a spatial resolution of $64 \times 64$ pixels and a size of $80 \times 80 \mathrm{~mm}$. This sensor records 32 time slots, each integrating over 10 picoseconds (Fig. 7, bottom row). The scene is a diffuse plane located $60 \mathrm{~mm}$ away from the sensor and has the same size as the sensor, but a spatial resolution of $128 \times 128$ pixels. The top row shows reconstructions with varying levels of additive Gaussian sensor noise. 


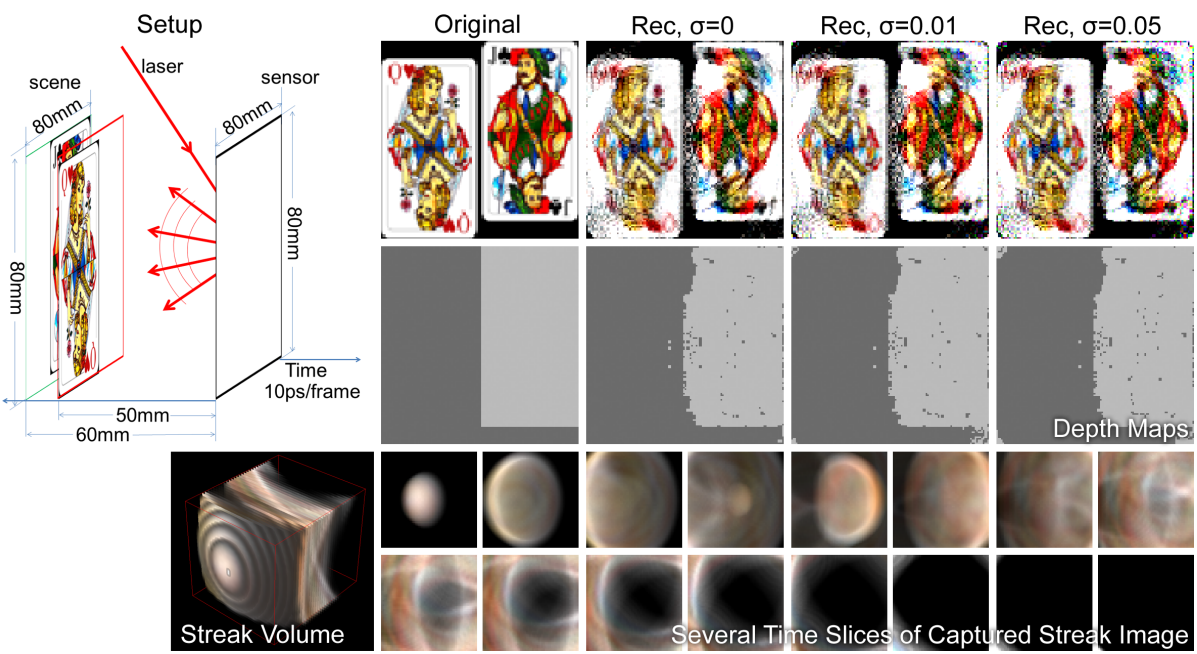

Fig. 8. Simulated results for reconstructing image and depth of an unknown scene captured with a bare sensor. Several time slices and a volumetric rendering of the recorded streak images are shown in the bottom row. While the scene depth can be reconstructed relatively accurately (center row), even small amounts of occlusion in the scene, as exhibited by the two playing cards, result in global noise in the reconstructed diffuse albedos (top row).

Fig. 8 shows simulated results for a scene with a varying depth. As discussed in Sec. 5, this requires the tomographic reconstruction to estimate both diffuse albedo and scene depth simultaneously. In this experiment, the spatial resolution of each of the depth planes is $96 \times 96$ pixels and the temporal sensor resolution is increased to measure 38 time slots with an exposure time of 10 picoseconds each. The reduced quality of these results can be entirely attributed to the occlusion between the cards in the scene. Even a small amount of occlusion, as exhibited in this experiment, results in a global increase in reconstruction noise.

Real results captured with a one-dimensional streak camera (see Sec. 6.2) are shown in Fig. 9. The intricate design of this camera prevents us from placing the bare sensor directly in the scene; we emulate this setup by recording a scene patch behind an optical diffuser instead. Challenges in this experiment are manifold: intensity variations and temporal jittering of the laser along with vignetting and non-linear temporal distortions in the streak camera result in variations of the recorded signal as compared to the predicted data using known scene geometry and albedo. Furthermore, a two-dimensional scene at a calibrated distance is measured with a one-dimensional sensor, which makes the reconstruction particularly difficult. Nevertheless, we show a successful reconstruction of a simple scene - a diffuse white patch - in Fig. 9, with errors due mainly to energy conservation constraints. 

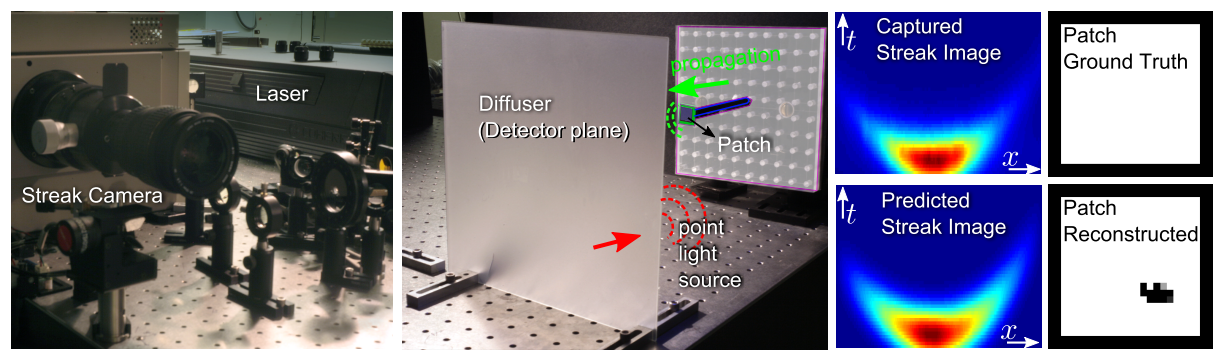

Fig. 9. The prototype camera setup shows the streak camera and the laser (left) and the scene (center left). Due to physical constraints, we model a bare sensor in space by imaging the light scattered onto an optical diffuser. Experimental and simulated streak images agree (center right). Succesful reconstruction of a $2 \mathrm{D}$ patch from a $1 \mathrm{D}$ sensor is shown on the right.

\subsection{Software Implementation}

We solve Eq. 14 using the simultaneous algebraic reconstruction technique (SART) [23]. This is an iterative approach to solve computed tomography problems. In our Matlab-based implementation, we run 10,000 iterations for each of the reconstructions in Fig. 7, 8, and 9. The large number of iterations is required due to the slow convergence rate of SART. The computations are performed with a sparse representation of matrix $\mathbf{P}$ in Eq. 14. Computing times are approximately one to two hours for all three color channels of each of the results.

\subsection{Hardware Setup}

For the captured result shown in Fig. 9, the illumination source is a femtosecond (fs) Titanium:Sapphire laser, which produced 50 fs long pulses, centered at 795 $\mathrm{nm}$ at a repetition rate of $75 \mathrm{MHz}$. The beam is focused onto the diffuser wall (ground glass). The streak camera is a Hamamatsu C5680, which is used to detect one spatial dimension of the diffuser with a time resolution of 15 picoseconds (ps) and quantum efficiency of about $10 \%$. The camera position and viewing direction are fixed.

\section{Conclusion}

In summary, we have presented a frequency analysis and a rigorous derivation of analytical expressions for the most fundamental process in light transportpropagation in free space, considering space, time, and angle. While prior models for the steady-state case intuitively describe light field propagation as a localized shear in both the primal and frequency domain, the hyperbolic curvature in space-time, introduced by differences in travel distances for different angles, delocalizes signal energy in the frequency domain. However, we demonstrate that it 
can be intuitively expressed as a combination of the well known shear in the light field and a convolution along the angular frequencies. This convolution optically blurs information between the different dimensions; we demonstrate that this information transfer can be computationally exploited using a novel, bare sensor imaging technique which has potential applications in biomedical imaging, thin mobile devices, remote sensing, and surveillance.

While the forward analysis presented is most general in considering space, time, and angle for arbitrary scenes, the inverse problem including bandwidth analysis and tomographic scene reconstruction restrict the imaged scene to be diffuse and to exhibit negligible amounts of global illumination effects and occlusions. The proposed sensing technique requires an ultra-short pulsed illumination, as for instance provided by a laser, and a streak camera capable of resolving time variations in the lower picosecond range. Currently available hardware achieving the required temporal resolution is restricted to sensing one spatial scanline per recorded image. The exposure time of the proposed system is significantly shorter than conventional imaging system; this fact in combination with the removed lens reduces the overall amount of light measured at each sensor pixel.

This work provides the fundmental analytic platfom on which to re-examine all computer vision applications in light of a new degree of freedom: time-offlight information. Further, it provides a method for building a consistent theory of time-resolved light field propagation. Future work will examine effects of optical elements other than propagation in free space, including lenses, diffusers, diffractive elements, and scattering media.

Acknowledgments. The work of the MIT affiliated coauthors was funded by the Media Lab Consortium Members, DARPA through the DARPA YFA grant, and the Institute for Soldier Nanotechnologies and U.S. Army Research Office under contract W911NF-07-D-0004. Gordon Wetzstein was supported by the DARPA SCENICC program. Tsinghua University affiliated coauthors were supported by China National Basic Research Project (No.2010CB731800) and the Key Project of NSFC (No. 61120106003 and 61035002).

\section{References}

1. Edgerton, H.E.: Electronic Flash, Strobe. MIT (1987)

2. Hamamatsu: Hamamatsu Streak Camera. http://learn.hamamatsu.com/ tutorials/java/streakcamera/ (2012)

3. Durand, F., Holzschuch, N., Soler, C., Chan, E., Sillion, F.X.: A Frequency Analysis of Light Transport. ACM Trans. Graph. (SIGGRAPH) 24 (2005) 1115-1126

4. Edward R. Dowski, J., Cathey, W.T.: Extended depth of field through wave-front coding. Applied Optics 34 (1995) 1859-1866

5. Levin, A., Hasinoff, S.W., Green, P., Durand, F., Freeman, W.T.: 4d frequency analysis of computational cameras for depth of field extension. ACM Trans. Graph. (Siggraph) 28 (2009) 97:1-97:14 
6. Veeraraghavan, A., Raskar, R., Agrawal, A., Mohan, A., Tumblin, J.: Dappled photography: Mask enhanced cameras for heterodyned light fields and coded aperture refocusing. ACM Trans. Graph. (SIGGRAPH) 26 (2007) 69

7. Ng, R.: Fourier slice photography. ACM Trans. Graph. (SIGGRAPH) 24 (2005) 735-744

8. Levin, A., Durand, F.: Linear view synthesis using a dimensionality gap light field prior. In: Proc. CVPR. (2010) 1-8

9. Raskar, R., Davis, J.: 5D Time-Light Transport Matrix: What Can We Reason about Scene Properties? MIT Technical Report (2008)

10. Zomet, A., Nayar, S.K.: Lensless imaging with a controllable aperture. In: Proc. CVPR. (2006) 339-346

11. Koppal, S.J., Gkioulekas, I., Zickler, T., Barrows, G.L.: Wide-angle micro sensors for vision on a tight budget. In: Proc. CVPR. (2011) 361-368

12. Andersen, G.: Large optical photon sieve. Opt. Lett. 30 (2005) 2976-2978

13. Gill, P.R., Lee, C., Lee, D.G., Wang, A., Molnar, A.: A microscale camera using direct fourier-domain scene capture. Opt. Lett. 36 (2011) 2949-2951

14. Saleh, B.: Introduction to Subsurface Imaging. Cambridge University Press (2011)

15. Velten, A., Willwacher, T., Gupta, O., Veeraraghavan, A., Bawendi, M., Raskar, R.: Recovering three-dimensional shape around a corner using ultra-fast time-offlight imaging. Nature Communications 3 (2012) 745-758

16. Naik, N., Zhao, S., Velten, A., Raskar, R., Bala, K.: Single view reflectance capture using multiplexed scattering and time-of-flight imaging. ACM Trans. Graph. (SIGGRAPH Asia) 30 (2011) 171

17. Pandharkar, R., Velten, A., Bardagjy, A., Lawson, E., Bawendi, M., Raskar, R.: Estimating motion and size of moving non-line-of-sight objects in cluttered environments. In: Proc. CVPR. (2011) 265-272

18. Wu, D., O'Toole, M., Velten, A., Agrawal, A., Raskar, R.: Decomposing global light transport using time of flight imaging. In: Proc. CVPR. (2012) 1-8

19. Isaksen, A., McMillan, L., Gortler, S.J.: Dynamically reparameterized light fields. ACM Trans. Graph. (SIGGRAPH) (2000) 297-306

20. Chai, J.X., Tong, X., Chan, S.C., Shum, H.Y.: Plenoptic sampling. ACM Trans. Graph. (SIGGRAPH) (2000) 307-318

21. Kajiya, J.T.: The Rendering Equation. ACM Trans. Graph. (SIGGRAPH) 20 (1986) 143-150

22. Smith, A., Skorupski, J., Davis, J.: Transient Rendering. Technical Report UCSCSOE-08-26, School of Engineering, University of California, Santa Cruz (2008)

23. Kak, A.C., Slaney, M.: Principles of Computerized Tomographic Imaging. Society for Industrial Mathematics (2001)

24. Herman, G.T.: Image reconstruction from projections. Real-Time Imaging 1 (1995) $3-18$

25. Chandrasekaran, V., Wakin, M., Baron, D., Baraniuk, R.: Surflets: A sparse representation for multidimensional functions containing smooth discontinuities. In: Information Theory, 2004. ISIT 2004. Proceedings. International Symposium on, IEEE (2004) 563 